\title{
Evaluation for estimating partition coefficients of alcohol tracer partitioning between benzene, toluene, ethylbenzene, and xylenes (BTEX) compounds and water
}

\author{
S. Rhee, S. Cho \& J. Park \\ School of Civil, Urban and Geosystem Engineering, \\ Seoul National University, Korea
}

\begin{abstract}
The partitioning tracer method has been studied in laboratory research and field investigations as an alternative method for characterizing aquifers contaminated by nonaqueous phase liquids (NAPLs). Accurate partition coefficients of tracers partitioning between NAPL and water are needed to improve the reliability of the partitioning tracer method. In this research, partition coefficients of alcohol tracers partitioning between benzene, toluene, ethylbenzene, and xylenes (BTEX) compounds and water are estimated using the approach of equivalent alkane carbon number (EACN). General agreement was observed between the measured and estimated partition coefficients of alcohol tracer between BTEX compounds and water. Based on these results we can verify that the EACN approach is suitable for estimating the partition coefficient of alcohol tracers between BTEX compounds and water.

Keywords: partitioning tracer method, alcohol tracers, partition coefficient, BTEX compounds.
\end{abstract}

\section{Introduction}

The contamination of soil and groundwater by nonaqueous phase liquids (NAPLs) has become an issue of great interest. In particular, petroleum, such as gasoline, has been used in large quantities in various industry field and its spills or leaks into aquifers cause environmental problems. The major components of petroleum are benzene, toluene, ethylbenzene, and xylenes (BTEX) and they 
have known to be carcinogenic organic contaminants [1]. If petroleum leaks into the ground from a storage tank it is necessary to monitor the distribution and quantity of petroleum and also to cleanup contaminants immediately [2].

However, traditional site characterization methods, such as analysis of soil gas, core sampling, and monitor-well sampling, are often not capable of providing sufficient data required for effective remediation system design. A major weakness of these methods is that they provide data at discrete points. As a result there is a probability of under-estimation of NAPL's residual saturation without a cost-prohibitive amount of sampling [3].

The partitioning tracer method has been developed and demonstrated in the field during the past few years as an alternative method for site characterization. The major advantage of the partitioning tracer method is to provide a continuous measurement of the target area and characterize a relatively large and reliable detection volume in comparison with traditional methods $[4,5]$. The partitioning tracer method consists of the simultaneous injection of several tracers, which have different partition coefficients with respect to NAPL at one or more injection wells and subsequent measurement of tracer concentrations at one or more extraction or monitoring wells. Injected tracers are retarded in proportion to its partition coefficient between NAPL and water due to their chromatographic separation in aquifers. This separation of the partitioning tracers indicates the presence of NAPL in the targeted zone and is used to determine the volume and distribution of NAPL present [4].

The accuracy of a partitioning tracer method mainly depends on the selection of suitable tracers and the accurate measurement of their partition coefficients between NAPL and water. Dwarakanath and Pope [5] suggest that the error in NAPL volume estimates based on partitioning tracer methods is directly proportional to the error in the partitioning coefficients. Therefore, the accurate experimental measurements of partition coefficients are important to minimize the errors in the partitioning tracer method. However, it requires time-consuming experiments to obtain the partition coefficient of each proposed tracer. Moreover, if samples of NAPL are not available, it is difficult to measure the partition coefficient directly.

Dwarakanath and Pope [5] proposed a new approach for estimating partition coefficients of alcohol tracers between NAPL and water using the concept of equivalent alkane carbon number (EACN), which is simply an equivalent of the alkane carbon numbers for more complex organic molecules (e.g., 6 for hexanol), and suggested that it is very effective and useful in estimating the partition coefficient of alcohol tracers between water and NAPLs. They also suggested the bilinear equation by multiple regression analysis on the basis of the results that the correlation between the logarithm of the partitioning coefficient of various alcohols and EACN of NAPLs and alcohol tracers is a linear relationship. Wu and Sabatini [6] have extended this approach and focused on more hydrophobic oils (high EACN LNAPLs) to find the hydrophobicity of NAPLs. These studies mainly focus on dense nonaqueous phase liquids (DNAPLs) and high EACN number of LNAPLs. 
In order to estimate the partition coefficient of alcohol tracers between petroleum and water by using EACN approach, BTEX are selected to represent gasoline as petroleum-contaminants and the EACN approach is applied for estimating the partition coefficients of several alcohol tracers for various BTEX mixtures. To confirm the accuracy of the EACN approach for petroleum, and in particular for gasoline, the partition coefficients from experimental measurements are compared with the estimated values from the EACN approach.

\section{Materials and methods}

\subsection{Materials}

The properties of benzene, toluene, ethylbenzene, and xylenes and alcohol tracers used in this study are summarized in table 1. BTEX mixtures are made with certain mixing ratios given in table 2. Mixtures $\mathrm{A}$ to $\mathrm{D}$ are blended for representing the mixtures with a certain mole ratio and Mixture E is blended with the special mixing ratio, which represent the composition ratio of BTEX found in soils contaminated by petroleum [7].

Table 1: $\quad$ Properties of BTEX and tracers used in this study.

\begin{tabular}{|c|c|c|c|c|c|c|}
\hline Chemicals & $\begin{array}{l}\text { Molecular } \\
\text { formula }\end{array}$ & $\begin{array}{c}\text { Molecular } \\
\text { weight }(\mathrm{g} / \mathrm{mol})\end{array}$ & $\begin{array}{l}\text { Density } \\
(\mathrm{g} / \mathrm{mL})\end{array}$ & $\begin{array}{l}\text { Solubility } \\
\text { (mg/L) }\end{array}$ & $\begin{array}{l}\text { Manufactured } \\
\text { company }\end{array}$ & $\begin{array}{l}\text { Purity } \\
(\%)\end{array}$ \\
\hline Benzene & $\mathrm{C}_{6}-\mathrm{H}_{6}$ & 78.11 & 0.88 & 1780 & JUNSEI & 99.5 \\
\hline Toluene & $\mathrm{C}_{6}-\mathrm{H}_{5}-\mathrm{C}-\mathrm{H}_{3}$ & 92.14 & 0.87 & 500 & Aldrich & 99.5 \\
\hline Ethylbenzene & $\mathrm{C}-\mathrm{H}_{3}-\mathrm{C}-\mathrm{H}_{2}-\mathrm{C}_{6}-\mathrm{H}_{5}$ & 106.17 & 0.86 & 140 & Aldrich & 98.0 \\
\hline Xylene & $\mathrm{C}_{6}-\mathrm{H}_{4}-\left(\mathrm{C}-\mathrm{H}_{3}\right)_{2}$ & 106.17 & 0.87 & 170 & Aldrich & 83.0 \\
\hline Methanol & $\mathrm{C}-\mathrm{H}_{3}-\mathrm{O}-\mathrm{H}$ & 32.04 & 0.79 & - & Duksan & 99.8 \\
\hline 1-Butanol & $\mathrm{C}_{4}-\mathrm{H}_{9}-\mathrm{O}-\mathrm{H}$ & 74.12 & 0.81 & - & Aldrich & 99.9 \\
\hline 1-Pentanol & $\mathrm{C}_{4}-\mathrm{H}_{9}-\mathrm{C}-\mathrm{H}_{2}-\mathrm{O}-\mathrm{H}$ & 88.15 & 0.81 & - & Aldrich & $99+$ \\
\hline Hexanol & $\mathrm{C}_{6}-\mathrm{H}_{14}-\mathrm{O}$ & 102.18 & 0.81 & - & Aldrich & $99+$ \\
\hline 2-Ethyl-1-Butanol & $\begin{array}{c}\left(\mathrm{C}_{2}-\mathrm{H}_{5}\right)_{2}-\mathrm{C}-\mathrm{H}-\mathrm{C}-\mathrm{H}_{2}- \\
\mathrm{O}-\mathrm{H}\end{array}$ & 102.18 & 0.83 & - & Aldrich & 98.0 \\
\hline $\begin{array}{c}\text { 4-Methyl-2- } \\
\text { Pentanol }\end{array}$ & $\begin{array}{c}\left(\mathrm{C}-\mathrm{H}_{3}\right)-\mathrm{C}-\mathrm{H}-\mathrm{C}-\mathrm{H}_{2}- \\
\mathrm{C}-\mathrm{H}-(\mathrm{O}-\mathrm{H})-\mathrm{C}-\mathrm{H}_{3}\end{array}$ & 102.18 & 0.81 & - & Aldrich & 99.0 \\
\hline
\end{tabular}

Table 2: $\quad$ Mixing ratios for BTEX compounds.

\begin{tabular}{c|cccc|cccc}
\hline \multirow{2}{*}{$\begin{array}{c}\text { BTEX } \\
\text { compounds }\end{array}$} & \multicolumn{9}{c}{ Mixing ratios } \\
\cline { 2 - 9 } & Benzene & Toluene & $\begin{array}{c}\text { Ethyl } \\
\text {-benzene }\end{array}$ & Xylene & Benzene & Toluene & $\begin{array}{c}\text { Ethyl } \\
\text {-benzene }\end{array}$ & Xylene \\
\hline Mixture A & 1 & 1 & 1 & 1 & 1 & 1.18 & 1.36 & 1.36 \\
Mixture B & 2 & 1 & 1 & 1 & 1 & 0.59 & 0.68 & 0.68 \\
Mixture C & 1 & 2 & 1 & 1 & 1 & 2.35 & 1.36 & 1.36 \\
Mixture D & 1 & 1 & 2 & 1 & 1 & 1.18 & 2.72 & 1.36 \\
Mixture E & 1 & 13.18 & 3.96 & 17.01 & 1 & 15.55 & 5.39 & 23.12 \\
\hline
\end{tabular}




\subsection{Experimental method}

The partition coefficient was measured by using six tracers and nine BTEX compounds, which were benzene, toluene, ethylbenzene, xylenes, and Mixture A to E. The alcohol aqueous concentrations were varied between 100 and 600 $\mathrm{mg} / \mathrm{L}(100,200,400,600 \mathrm{mg} / \mathrm{L})$. Equal volumes of BTEX compounds and water (10 mL each) were placed in a $20 \mathrm{~mL}$ vial that was crimp-capped with a Teflon septum, leaving minimum headspace. The vial was shaken on a vortex mixer and rotary shaker for each 30 seconds and 24 hours. After shaking these samples, they were allowed to stabilize for 1 hour and than centrifuged for 5 minutes to allow a complete separation of the phases. Reacted alcohol aqueous solution was translated from aqueous phase to the $2 \mathrm{~mL}$ vial using a pipette and were analyzed by an Agilent 6890 GC-FID (each sample was made in duplicate). Then, the partition coefficient was calculated from using a mass balance.

\section{Results and discussions}

The partitioning of 4-methyl-2-pentanol between BTEX compounds and water is shown in fig. 1. Linear relationships are found between the concentration of a tracer in water and that of a tracer in BTEX compounds. The other 4 tracers, which are 1-butanol, 1-pentanol, hexanol, and 2-ethyl-1-butanol, also show linear relationships between the concentration of a tracer in water and that of a tracer in BTEX compounds.

From these linear relationships the slope of the regression curve represents the partition coefficient of used alcohol tracer. All of partition coefficients for each alcohol tracer between BTEX compounds and water are listed in table 3 and the regression coefficient of results have a value of over 0.95 .

The EACN values of benzene, toluene, ethylbenzene, and toluene are respectively $0,1,2$, and 2 and those of butanol, pentanol, and hexanol are respectively 4, 5, and 6. BTEX's EACN values are from the literature $[8,9]$ and the tracer's EACN values are from the assumption that the EACN value of linear alcohol can be found just by its carbon number [5].

A linear relationship was observed when the logarithm of the partition coefficient of several alcohol tracers was plotted against both of the EACN values of BTEX compounds (fig. 2) and those alcohol tracers (fig. 3). These results are similar with that of Dwarakanath and Pope [5].

According to these results, the bilinear equation for correlating the logarithm of partition coefficient (K) as a function of the alcohol tracer's EACN (A) and the BTEX EACN $(\mathrm{N})$ was obtained by multiple regression analysis, eqn (1).

$$
\log K=-2.39829+0.584492 \cdot A-0.07418 \cdot N
$$

Based on the regression of the data, the standard deviation is $15.5 \%$ and the correlation coefficient of the regression is 0.935. This equation for BTEX compounds is analogous to that of Dwarakanath and Pope [5]. 
Using the experimental value of the partition coefficient for single BTEX and eqn. (1), the logarithm of the experimental partition coefficient of the BTEX mixture is compared with the logarithm of the estimated partition coefficient of the BTEX mixtures (table 4) and the accuracy of measurements is given in percent (estimated value over experimental value times 100). The overall difference and tendency between the experimental and estimated results is also plotted in fig. 4 . On the 1:1 line, experimental values are equal to estimated value and most over-estimated or under-estimated values are on the 1:1.19 line and 1:0.58 line respectively. Though there is a tendency that estimated results are underestimated, most of the estimated results are similar to experimental results.

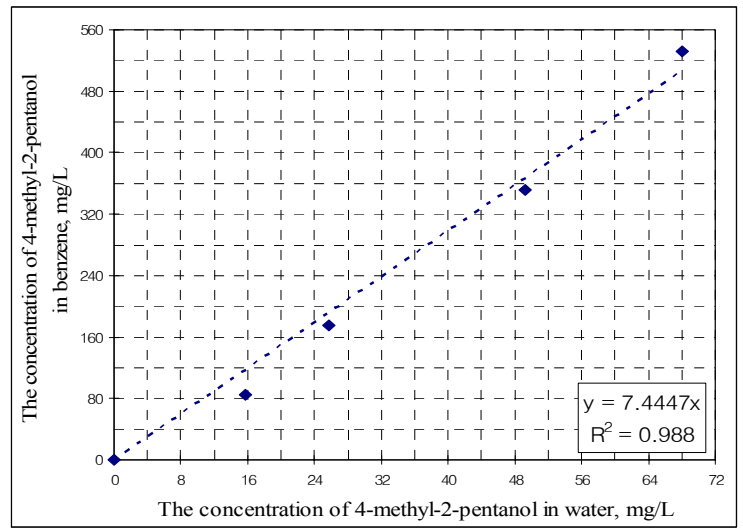

(a)

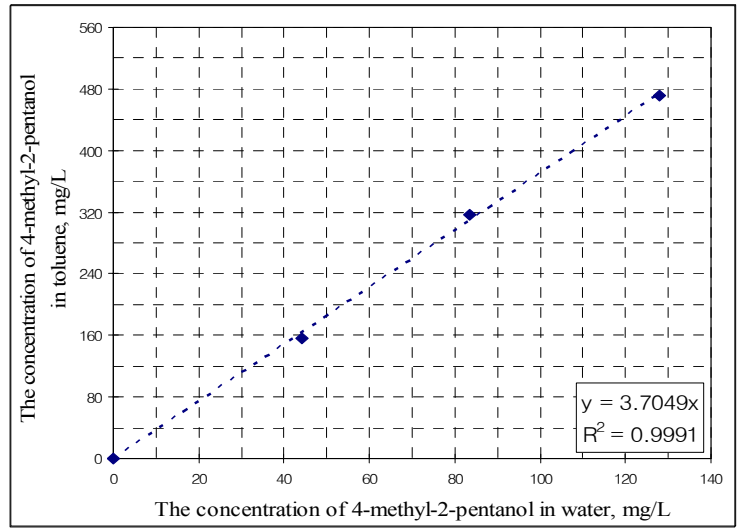

(b)

Figure 1: Partitioning of 4-methyl-2-pentanol between water and: (a) benzene, (b) toluene, (c) Mixture A and (d) Mixture B. 


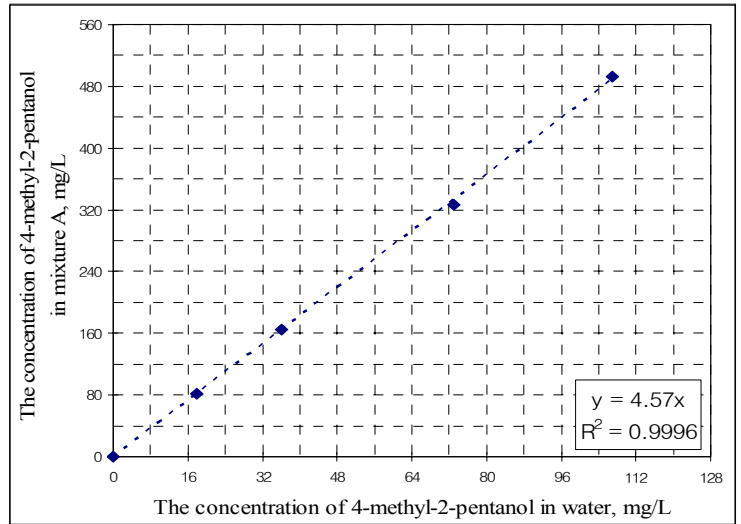

(c)

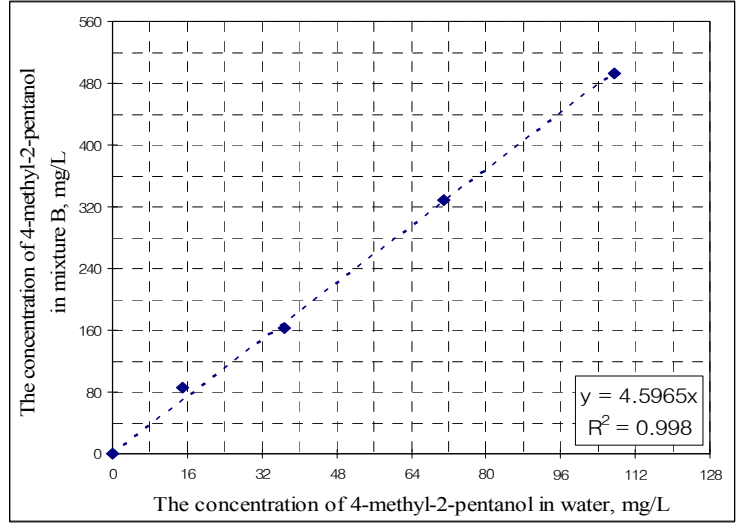

(d)

Figure 1: Continued

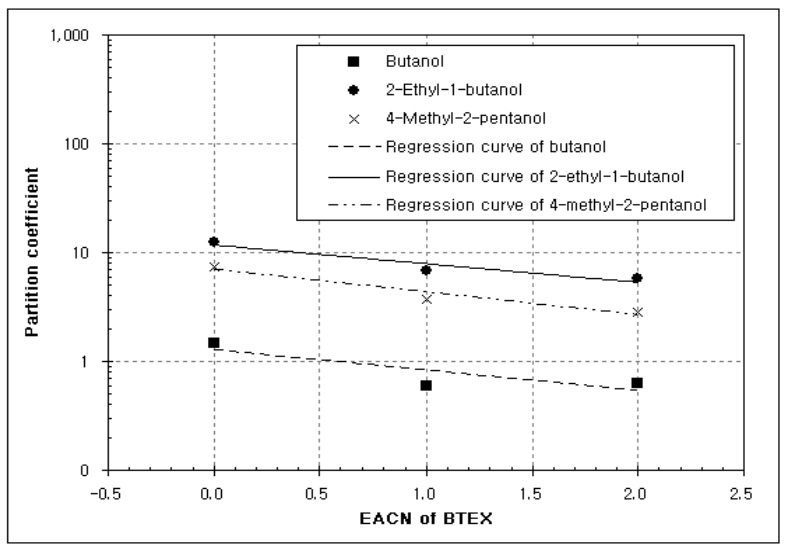

Figure 2: Partitioning coefficient as a function of BTEX EACN. 
Table 3: Measured partition coefficients of tracers between BTEX compounds and water.

\begin{tabular}{|c|c|c|c|c|c|c|}
\hline Tracers & Contaminants & $\mathrm{K}^{a}$ & $\mathrm{R}^{2 b}$ & Contaminants & $\mathrm{K}$ & $\mathrm{R}^{2}$ \\
\hline \multirow{5}{*}{ Methanol } & Benzene & 0.05 & - & Mixture A & - & - \\
\hline & Toluene & 0.22 & - & Mixture B & - & - \\
\hline & & & & Mixture $\mathrm{C}$ & - & - \\
\hline & Ethylbenzene & 0.28 & - & Mixture D & - & - \\
\hline & Xylene & 0.19 & - & Mixture E & - & - \\
\hline \multirow{5}{*}{ Butanol } & Benzene & 1.47 & 0.9999 & Mixture A & 1.99 & 0.9745 \\
\hline & Toluene & 0.60 & 0.9784 & Mixture B & 1.10 & 0.9955 \\
\hline & & & & Mixture $\mathrm{C}$ & 0.76 & 0.9984 \\
\hline & Ethylbenzene & 0.62 & 0.9992 & Mixture D & 0.96 & 0.9719 \\
\hline & Xylene & 0.88 & 0.9429 & Mixture E & 0.42 & 0.9983 \\
\hline \multirow{5}{*}{ Pentanol } & Benzene & 2.68 & 0.9793 & Mixture A & 2.36 & 0.9922 \\
\hline & Toluene & 1.98 & 0.9918 & Mixture B & 2.49 & 0.9994 \\
\hline & & & & Mixture C & 2.31 & 0.9988 \\
\hline & Ethylbenzene & 2.20 & 0.9915 & Mixture D & 2.37 & 0.9905 \\
\hline & Xylene & 1.68 & 0.9990 & Mixture E & 2.88 & 0.9480 \\
\hline \multirow{5}{*}{ Hexanol } & Benzene & 14.00 & 0.9954 & Mixture A & 14.48 & 0.9679 \\
\hline & Toluene & 8.65 & 0.9900 & Mixture B & 10.67 & 0.9979 \\
\hline & Ethylbenzene & 14.51 & 0.9931 & Mixture C & 7.62 & 0.9952 \\
\hline & Xvlene & 12.06 & 09963 & Mixture D & 18.23 & 0.9579 \\
\hline & & & & Mixture E & 19.29 & 0.9643 \\
\hline \multirow{4}{*}{ 2-Ethyl-1-butanol } & Benzene & 12.59 & 0.9885 & Mixture A & 6.97 & 0.9996 \\
\hline & Toluene & 6.86 & 0.9903 & Mixture B & 6.74 & 0.9879 \\
\hline & Ethylbenzene & 5.71 & 1.0000 & $\begin{array}{l}\text { Mixture C } \\
\text { Mixture D }\end{array}$ & 8.07 & 0.9851 \\
\hline & Xylene & 5.95 & 0.9995 & $\begin{array}{l}\text { Mixture D } \\
\text { Mixture E }\end{array}$ & $\begin{array}{l}6.56 \\
5.74\end{array}$ & $\begin{array}{l}0.9918 \\
0.9902\end{array}$ \\
\hline \multirow{5}{*}{ 4-Methyl-2-pentanol } & Benzene & 7.44 & 0.9880 & Mixture A & 4.57 & 0.9996 \\
\hline & Toluene & 3.70 & 0.9991 & Mixture B & 4.60 & 0.9980 \\
\hline & Ethylbenzene & 283 & 0.9899 & Mixture $\mathrm{C}$ & 6.15 & 0.9752 \\
\hline & & & 0.9899 & Mixture D & 4.55 & 0.9883 \\
\hline & Xylene & 3.48 & 0.9951 & Mixture E & 9.18 & 0.9488 \\
\hline
\end{tabular}

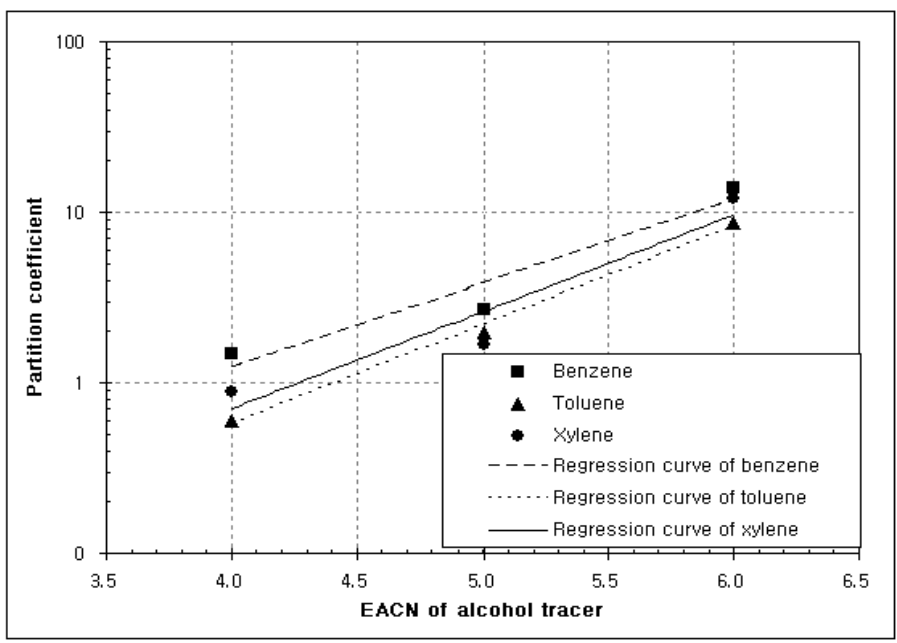

Figure 3: $\quad$ Partitioning coefficient as a function of alcohol EACN. 
Table 4: $\quad$ Measured and estimated partition coefficients of alcohol tracers.

\begin{tabular}{ccccc}
\hline Tracers & Mixtures & Exp. logK & Est. logK & Accuracy(\%) \\
\hline \multirow{5}{*}{ Pentanol } & Mixture A & 0.3729 & 0.3231 & 86.65 \\
& Mixture B & 0.3962 & 0.3441 & 86.86 \\
& Mixture C & 0.3636 & 0.3178 & 87.41 \\
& Mixture D & 0.3747 & 0.3270 & 87.26 \\
& Mixture E & 0.4594 & 0.2727 & 59.36 \\
\hline \multirow{5}{*}{ Hexanol } & Mixture A & 1.1608 & 1.0815 & 93.17 \\
& Mixture B & 1.0282 & 1.0945 & 106.45 \\
& Mixture C & 0.8820 & 1.0526 & 119.35 \\
& Mixture D & 1.2608 & 1.0976 & 87.05 \\
& Mixture E & 1.2852 & 1.0394 & 80.87 \\
\hline \multirow{5}{*}{ 2-Ethyl-1-butanol } & Mixture A & 0.8432 & 0.8669 & 102.80 \\
& Mixture B & 0.8287 & 0.9135 & 110.24 \\
& Mixture C & 0.9069 & 0.8608 & 94.92 \\
& Mixture D & 0.8169 & 0.8448 & 103.42 \\
& Mixture E & 0.7589 & 0.8084 & 106.53 \\
\hline & Mixture A & 0.6599 & 0.6083 & 92.18 \\
& Mixture B & 0.6628 & 0.6609 & 99.73 \\
& Mixture C & 0.7889 & 0.6003 & 76.09 \\
& Mixture D & 0.6580 & 0.5770 & 87.69 \\
& Mixture E & 0.9628 & 0.5548 & 57.62 \\
\hline
\end{tabular}

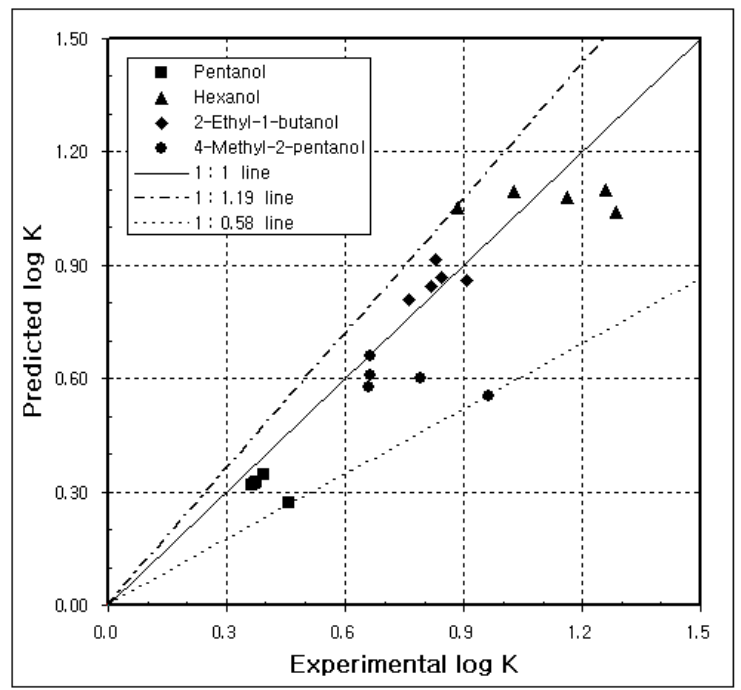

Figure 4: Comparison of measured and estimated partition coefficients of tracers.

\section{Summary and conclusion}

The linear relation between the concentration of the tracer in water and that of the tracer in BTEX compounds was confirmed with high accuracy (regression 
coefficients are over 0.95) and partition coefficients of alcohol tracers were obtained under this linear relation.

The bilinear relation was found for correlating the logarithm of the partition coefficient for alcohol tracer partitioning between BTEX compounds and water as a function of the alcohol tracer's EACN and the BTEX EACN. As a result of comparison between experimental and estimated partition coefficients, a general agreement was observed although there is a tendency that the estimated results are underestimated.

Therefore, we can conclude that the EACN approach can be extended to estimate the partition coefficient for alcohol tracer partitioning between BTEX compounds and water.

\section{Acknowledgements}

This subject is supported by Korea Ministry of Environment as "The Ecotechnopia 21 project".

\section{References}

[1] Schade, W. \& Bublitz, J. On-site laser probe for the detection of petroleum products in water and soil, Environ. Sci. Technol., 30(5), pp. 1451 1458, 1996.

[2] Bachman, J., Kanan, S. M. \& Patterson, H. H. Monitoring laboratory-scale bioventing using synchronous scan fluorescence spectroscopy: analysis of vapor phase, Environmental pollution, 113, pp. 155 162, 2001.

[3] Brusseau, M. L., Nelson, N. T., \& Cain, R. B. The partitioning tracer method for In-situ detection and quantification of immiscible liquids in the subsurface, ACS symposium series 725, 1999

[4] Jin, M., Delshad, M., Dwarakanath, V., McKinney, D. C., Pope, G. A., Sepehrnoori, K., \& Tilburg, C. E. Partitioning tracer test for detection, estimation, and remediation performance assessment of subsurface nonaqueous phase liquids, Water Resources Research, 31(5), pp. 12011211 , 1995.

[5] Dwarakanath, V. \& Pope, G. A. New approach for estimating alcohol partition coefficients between nonaqueous phase liquids and water, Environ. Sci. Technol., 32, pp. 1662 1666, 1998.

[6] Wu, B. \& Sabatini, D. A. Using partitioning alcohol tracers to estimate hydrophobicity of high molecular weight LNAPLs, Environ. Sci. Technol., 34, pp. $47014707,2000$.

[7] Park, H. M., Kim, J. H. Kim, Y. M., \& Lee, K. B. BTEX contamination level of soil samples around gas stations in Korea, Journal of the Korea Society for Environmental Analysis, 4, pp. 223 227, 2000.

[8] Cayias, J.L., Schechter, R.S., \& Wade, W.H., Modeling Crude Oils for Low Interfacial Tension, Society of Petroleum Engineers Journal, 16, pp. 351 $357,1976$. 
260 Brownfields III

[9] Baran, J. R., Jr., Pope, G. A., Wade, W. H., Weerasooriya, V., \& Yapa, A. Microemulsion formation with mixed chlorinated hydrocarbon liquids, Journal of Colloid and Interface Science, 4, pp. 223 227, 1994. 